\title{
Optimización del Proceso de Extracción de Antocianinas y Evaluación de la Capacidad Antioxidante de Berenjena (Solana melonera L.)
}

\author{
Irina Heras, Armando Alvis y Guillermo Arrazola \\ Universidad de Córdoba, Programa de Ingeniería de Alimentos, Facultad de Ingenierías, \\ Grupo de Investigación Procesos y Agroindustria de vegetales, Carrera 6 No76-103, Km 3, vía Cereté. \\ Córdoba - Colombia. (e-mail: guillermo.arrazola@ua.es)
}

Recibido Mar. 18, 2013; Aceptado Abr. 17, 2013; Versión final recibida May. 26, 2013

\begin{abstract}
Resumen
El objetivo de esta investigación fue optimizar el proceso de extracción de antocianinas obtenidas de berenjena (Solanum melongena L.) y evaluar su capacidad antioxidante. A extractos de piel y pulpa se le realizaron análisis fisicoquímicos, contenido en fenoles y antocianos. Para la optimización del proceso de extracción de las antocianinas se empleó la metodología de superficie de respuesta con tres factores: concentración de solvente etanol acidificado 1\% ácido ortofosfórico (50 a 90\% v/v), temperatura extracción $\left(30\right.$ a $60^{\circ} \mathrm{C}$ ) y tiempo extracción (4 a 12 horas). La evaluación de la capacidad antioxidante se realizó empleando el método ABTS (6-sulfonato-3-etilbenzotiazolina). El proceso de extracción de antocianinas de la cáscara sigue un modelo cuadrático, mientras que la capacidad antioxidante tuvo un comportamiento lineal. La optimización del modelo mostró que las condiciones de extracción con $50 \%$ de solvente acidificado, tiempo de proceso de 4 horas y temperatura de $30{ }^{\circ} \mathrm{C}$ presentó los mayores contenidos de antocianinas, alcanzando $62 \mathrm{mg} / 100 \mathrm{~g}$.
\end{abstract}

\section{Optimization of the Anthocyanin Extraction Process and Evaluation of the Antioxidant Capacity of Eggplant (Solanum melongena $L$.)}

\begin{abstract}
The objective of this research was to optimize the extraction of anthocyanins obtained from eggplant (Solanum melongena L.) and to evaluate their antioxidant capacity. Physicochemical analyses, phenols content and anthocyanins content were performed in skin and pulp extracts. To optimize the extraction process anthocyanins response surface methodology with three factors was employed: acidified ethanol concentration of solvent $1 \%$ orthophosphoric acid (50 to $90 \% \mathrm{v} / \mathrm{v})$, extraction temperature $\left(30\right.$ to $60{ }^{\circ} \mathrm{C}$ ) and extraction time (4 to 12 hours). The antioxidant capacity evaluation was performed using the ABTS method (6-ethylbenzothiazoline-3-sulfonate). The extraction process of anthocyanins from the skin followed a quadratic model, while the antioxidant capacity followed a linear behavior. The optimization of the model showed that extraction with $50 \%$ acidified solvent, process time of 4 hours and temperature of $30{ }^{\circ} \mathrm{C}$ presented the highest anthocyanins content, reaching $62 \mathrm{mg} / 100 \mathrm{~g}$.
\end{abstract}

Keywords: anthocyanins, antioxidant activity, eggplant, Solanum melongena L., total phenols 


\section{INTRODUCCIÓN}

Las antocianinas son compuestos fenólicos, de la familia de los flavonoides, colorantes naturales ampliamente distribuidos en frutas, bayas y flores, ofreciendo atractivos colores, como naranja, rojo y azul. Estos pigmentos son solubles en agua y esta propiedad facilita su incorporación en numerosos sistemas alimentarios acuosos. Estas cualidades hacen que las antocianinas sean colorantes naturales atractivos (Kong et al., 2003). El interés por los pigmentos antociánicos en investigaciones científicas se han incrementado en los últimos años, debido no sólo al color que confieren a los productos que las contienen sino en la reducción de las enfermedades coronarias, cáncer, diabetes, efectos antiinflamatorios, mejoramiento de la agudeza visual y comportamiento cognitivo; estos efectos terapéuticos positivos, están principalmente asociados con sus propiedades antioxidantes (Kong et al., 2003; Longo y Vasapollo, 2006; Garzón, 2008; Todaro et al., 2009). Además por su gran potencial para el reemplazo competitivo de colorantes sintéticos; es de gran importancia conocer los aspectos bioquímicos que enmarcan estos pigmentos (Garzón, 2008, Hongyan et al., 2012).

La berenjena contiene ácido ascórbico y compuestos fenólicos que le confieren un gran poder antioxidante, principalmente asociado a algunos efectos terapéuticos positivos (Noda et al., 2000; Nisha et al., 2009; Todaro et al., 2009; Singh et al., 2009). Se encuentra entre una de las hortalizas con capacidad de absorción de radicales de oxígeno (Botelho et al., 2004). El objetivo de esta investigación fue extraer, cuantificar y optimizar el proceso de extracción de antocianinas a partir de cascara de berenjena (Solanum melongena) y evaluar su capacidad antioxidante.

\section{MATERIALES Y MÉTODOS}

Los reactivos que se utilizaron, fueron, el hidróxido de sodio, ácido clorhídrico, Trolox (6-hidroxi-2,5,7,8tetrametilcromo- 2-ácido carboxílico 97\%,) como antioxidante de referencia, ABTS, 2,2'azinobis (3etilbenzotiazolin 6-ácido sulfónico) en forma de sal diamónica, persulfato potásico de Sigma Aldrich, Carbonato de sodio, Reactivo de Folin Ciocalteau y acido gálico (Merck). El material vegetal recolectado fueron de berenjenas de los cultivares fucsia y lila campana cultivada en el departamento de Córdoba. La recolección de las berenjenas se realizó en la fase de maduración comercial. Los frutos de berenjena fueron lavados y almacenados a temperaturas de $4^{\circ} \mathrm{C}$.

\section{Caracterización fisicoquímica de berenjenas}

La extracción de antocianinas se realizó en la pulpa (100 mg) y en la piel o cáscara de berenjena (100 mg). Después de la recolección de la hortaliza, las muestras fueron peladas y la pulpa fue separada de la cáscara, empleando un cuchillo de acero inoxidable. Se extrajo capas de cáscara de berenjena con un espesor de $1 \mathrm{~mm}$, y fueron cortadas para obtener un tamaño de partícula de $20 \mathrm{~mm}^{2}$ aproximadamente (Todaro et al., 2009). Se realizó la determinación de pH empleando un pH- metro Schott modelo lab 850; análisis de acidez, expresado como porcentaje de ácido cítrico; la medición de sólidos solubles totales ( ${ }^{\circ}$ Brix) se realizó con un refractómetro digital Atago model Pal- $\alpha$. El contenido de fenoles totales se determinó por el método de Folin-Ciocalteau Severo et al.(2009), realizando una curva patrón con ácido gálico midiendo lecturas en un espectrofotómetro (Génesis 20) a $765 \mathrm{~nm}$. Se realizó la determinación del total de monómeros de antocianinas por el método diferencial de pH descrito por (Giusti y Wrolstad, 2001; Poo, 2005). El contenido de pigmentos en el extracto se calculó en $\mathrm{mg} / 100 \mathrm{~g}$ de cianidina 3- glucosido. El extracto fue diluido con tampón de $\mathrm{pH}$ de 1 y pH de 4,5 y se midió la absorbancia a $520 \mathrm{~nm}$ (Iongitud de onda máxima) y a $700 \mathrm{~nm}$. El factor de dilución fue el mismo para ambas muestras $(\mathrm{pH} 1,0$ y pH 4,5) (Giusti y Wrolstad, 2001; Poo, 2005).

\section{Extracción de antocianinas.}

Para la extracción de las antocianinas de berenjena $(100 \mathrm{mg})$ se utilizó las siguientes condiciones de extracción: se empleó como solvente etanol acidificado con ácido ortofosfórico, (etanol 50-90\% v/v), temperatura de extracción $\left(30\right.$ a $60^{\circ} \mathrm{C}$ ) y tiempo de extracción (4 a 12 horas) (Todaro et al., 2009; Martínez, 2009). Los ensayos se llevaron cabo en frascos de color ámbar en ambientes oscuros. La mezcla fue filtrada en un embudo Buchner y el sólido recuperado fue lavado con el solvente etanol acidificado hasta obtener una solución clara. El sobrenadante de las filtraciones se combinó y luego fueron concentrados en un rotaevaporador a $42{ }^{\circ} \mathrm{C}$ (Longo y Vasapollo 2006). La solución concentrada de antocianinas fue almacenada a $4{ }^{\circ} \mathrm{C}$ en frascos de color ámbar. Los extractos de antocianinas fueron sometidos a liofilización empleando un equipo liofilizador marca LABCONCO Freezone 1 , operado a $-42{ }^{\circ} \mathrm{C}$, y $0.013 \mathrm{mBar}$ durante un periodo de 24 horas. 


\section{Determinación de poder antioxidante.}

La actividad antioxidante de los extractos se midió empleando el método ABTS (6-sulfonato-3etilbenzotiazolina), las mediciones de absorbancia se realizaron a $732 \mathrm{~nm}$ empleando un espectrofotómetro (Genesis 20). El radical ABTS se generó por la reacción de oxidación del ABTS (2,2'-azino-bis-(3etilbenzotiazolin-6-sulfonato de amonio)) $(3,5 \mathrm{mM})$ con persulfato de potasio $(1,25 \mathrm{mM})$. Después de $24 \mathrm{~h}$ de reacción, se diluyó el reactivo ABTS concentrado con solución tampón fosfato a pH 7,4 hasta absorbancia 0,70 , a una longitud de onda de $732 \mathrm{~nm}$. Para la evaluación se emplearon $10 \mu \mathrm{L}$ del extracto diluido en DMSO (Dimetilsufoxido) y $990 \mu \mathrm{L}$ de la solución del radical ABTS+. Luego de 30 min de reacción a temperatura ambiente y en la oscuridad, se leyó el cambio en la absorbancia respecto a la referencia del reactivo. Se midió también el blanco y la referencia del solvente y la muestra en iguales condiciones. Los resultados se expresaron como IC50 (Porcentaje de inhibición de la muestra) y como valores TEAC mediante la construcción de una curva patrón usando diferentes concentraciones de TROLOX® (ácido 6hidroxi-2,5,7,8-tetrametil-2-cromanocarboxílico). (Trejo y Bustamante, 2002).

\section{Diseño experimental y análisis de datos}

Para el análisis del contenido de antocianinas de berenjena se empleó la metodología de superficie de respuesta, haciendo uso del software estadístico comercial Desing Expert Versión 6.0.1 (Stat-Ease, Minneapolis, USA) para generar el diseño experimental, los análisis estadísticos y el modelo de regresión. Se empleó un diseño factorial a tres niveles. Tres variables independientes fueron analizadas a saber, concentración de solvente etanol acidificado (Etanol 50-90\% v/v), temperatura de extracción $\left(30\right.$ a $\left.60^{\circ} \mathrm{C}\right)$ y tiempo de extracción (4 a 12 horas). Un total de 32 diferentes combinaciones (incluyendo seis réplicas del punto central) fueron seleccionadas en orden aleatorio de acuerdo a la configuración del diseño para los tres factores. Las variables respuestas fueron concentración de compuestos fenólicos, contenido de antocianinas y capacidad antioxidante de las muestras.

\section{RESULTADOS Y DISCUSIÓN}

Los resultados de la caracterización fisicoquímica de la berenjena proveniente de cultivos de productores del municipio de Retiro de los Indios se muestran en la Tabla 1.

Tabla 1. Análisis Fisicoquímicos de Pulpa de Berenjena

\begin{tabular}{lll}
\hline Parámetro & Berenjena Morada & Berenjena Lila \\
\hline $\mathrm{pH}$ & $5,59 \pm 0.05 \mathrm{a}$ & $5,47 \pm 0.01 \mathrm{~b}$ \\
Acidez (\% ácido cítrico) & $0,14 \pm 0.02 \mathrm{a}$ & $0,2 \pm 0.02 \mathrm{~b}$ \\
Sólidos Solubles (\%) & $4,39 \pm 0.06 \mathrm{a}$ & $4,83 \pm 0.03 \mathrm{~b}$ \\
Fenoles Totales (\%)BS & $29.72 \pm 1^{*} \mathrm{a}$ & $62,5 \pm 0.7 \mathrm{~b}$ \\
Antocianinas (\%)BS & $2 \pm 0.2^{*} \mathrm{a}$ & $2,78 \pm 0.02 \mathrm{~b}$ \\
\hline
\end{tabular}

Los valores medios de las todas las variables fisicoquímicas en estudio presentaron diferencias significativas $(p<0,05)$, en pulpas de berenjenas fucsia- morada y Lila, observándose que los contenidos de fenoles totales, antocianinas, acidez y sólidos solubles fueron mayores en berenjena lila.

Por otro lado en la tabla 1 , se puede observar el contenido de fenoles totales de berenjena fucsia-morada y lila, los cuales fueron de 29.72 y $62,5 \mathrm{mg}$ equivalentes de ácido $/ 100 \mathrm{~g}$ de pulpa fresca $\left(3,8 \mathrm{mg} \mathrm{g}^{-1} \mathrm{y} 8 \mathrm{mg} \mathrm{g}^{-1}\right.$ en morada negra y lila en base seca); el contenido de fenoles se obtuvo a partir de la curva patrón de ácido gálico a través de la técnica de Folin Ciocalteu. Asimismo el contenido de antocianinas en pulpa de berenjena Morada y Lila en este trabajo fue de $2 \mathrm{mg} / 100 \mathrm{~g}$ y $2,78 \mathrm{mg} / 100 \mathrm{~g}$.

\section{Extracción de antocianinas a partir de cáscara de berenjenas}

En el proceso de extracción de antocianinas de la cascara de berenjena, se observó que el modelo que explica significativamente $(p<0,05)$ el contenido de antocianinas respecto a las variables estudio, concentración de solvente, tiempo y temperatura de extracción fue un modelo cuadrático. Los resultados de la Anava (Tabla 2) muestran que las variables concentración de solvente, tiempo y temperatura de extracción fueron significativas en la extracción de antocianinas. El coeficiente cuadrático de la temperatura es significativo para el contenido de antocianinas. Sin embargo las interacciones de las variables en estudio no fueron significativas $(p<0.05)$. 
El valor del coeficiente de determinación $\left(R^{2}\right)$ fue de 0,9169 indicando que el modelo obtenido es capaz de explicar el $91,69 \%$ de las variaciones en los contenidos de antocianinas. El modelo ajustado al contenido de antocianos presentó la siguiente ecuación, con t= tiempo, $T=$ temperatura y Sol=concentración de solvente

Contenido antocianinas $=64.67-1.3 \mathrm{t}+2.05 \mathrm{~T}-0.77 \mathrm{Sol}-0,036 \mathrm{~T}^{2}$

En la figura 1a se puede observar que la concentración de antocianinas disminuye con el aumento de la temperatura de extracción. Con respecto a la concentración de solvente y el tiempo de extracción, se observa un aumento del contenido de antocianinas cuando estas dos variables aumentan figura $1 \mathrm{~b}$; $\sin$ embargo en el rango de estudio no se vio un efecto cuadrático significativo.

Tabla 2. Análisis de Varianza de contenido de antocianinas. En la Tabla $A=$ Tiempo; $B=$ Temperatura; $C=$ Concentración de solvente; $\mathrm{S}$ = significante; $\mathrm{NS}$ = no significante.

\begin{tabular}{crrrrcc}
\hline Fuente & Suma De Cuadrados & Df & Cuadrados Medios & F Value & Prob $>$ F & Significancia \\
\hline Modelo & 5003,94 & 9 & 555,99 & 26,96 & $<0.0001$ & $\mathrm{~S}$ \\
A & 115,01 & 1 & 115,01 & 5,58 & 0.0275 & $\mathrm{~S}$ \\
B & 4215,46 & 1 & 4215,46 & 204,38 & $<0.0001$ & $\mathrm{~S}$ \\
C & 127,63 & 1 & 127,63 & 6,19 & 0.0209 & $\mathrm{~S}$ \\
$\mathrm{~A}^{2}$ & 0,00 & 1 & 0,00 & 0,00 & 0.9953 & $\mathrm{NS}$ \\
$\mathrm{B}^{2}$ & 483,00 & 1 & 483,00 & 23,42 & $<0.0001$ & $\mathrm{~S}$ \\
$\mathrm{C}^{2}$ & 6,84 & 1 & 6,84 & 0,33 & 0.5704 & $\mathrm{NS}$ \\
AB & 2,61 & 1 & 2,61 & 0,13 & 0.7253 & $\mathrm{NS}$ \\
AC & 16,76 & 1 & 16,76 & 0,81 & 0.3772 & $\mathrm{NS}$ \\
BC & 17,11 & 1 & 17,11 & 0,83 & 0.3722 & $\mathrm{NS}$ \\
Residual & 453,76 & 22 & 20,63 & & & \\
Ajustes & 219,33 & 17 & 12,90 & 0,28 & 0.9797 & $\mathrm{NS}$ \\
Error & 234,43 & 5 & 46,89 & & & \\
Correlación Total & 5457,70 & 31 & & & & \\
\hline
\end{tabular}
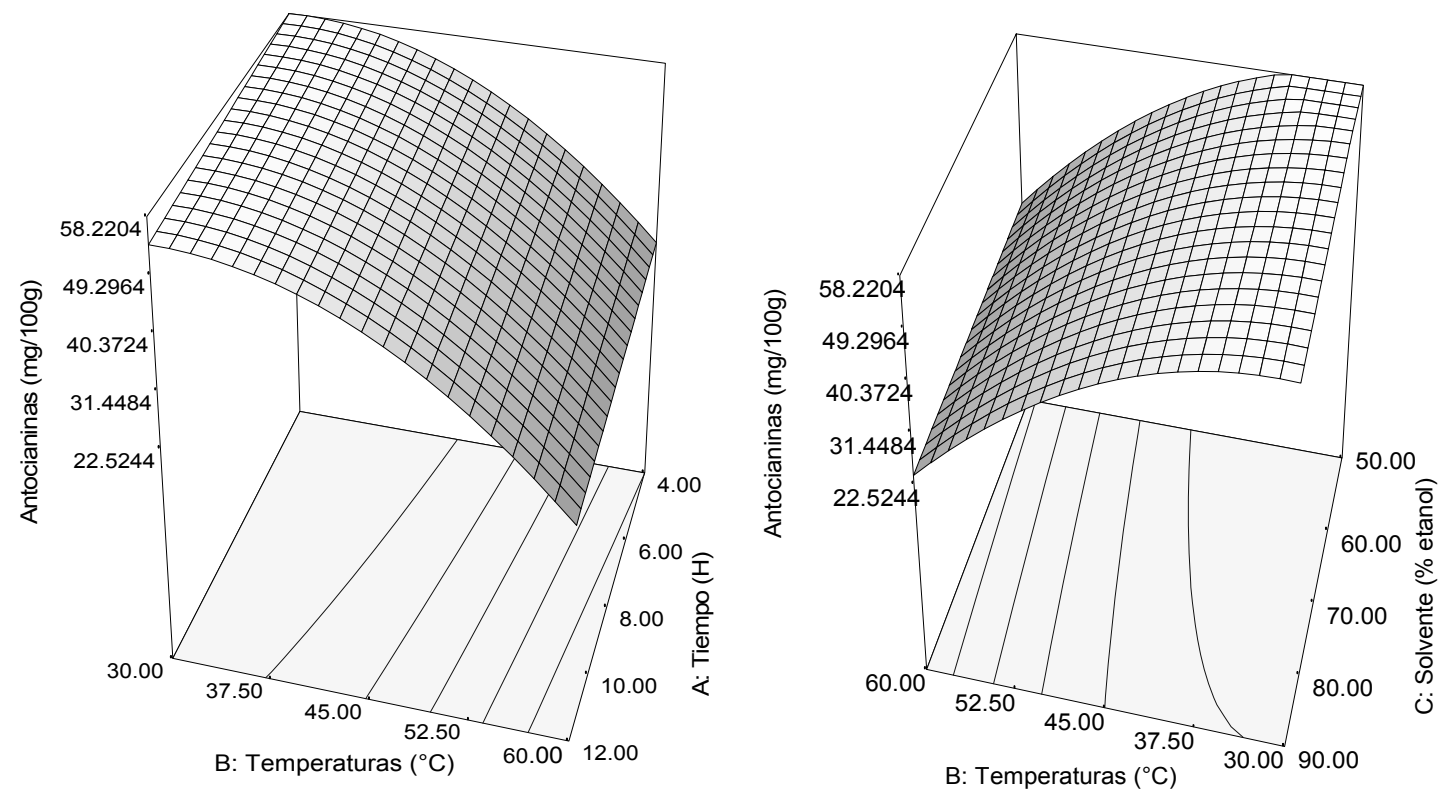

Fig. 1. Superficie de respuesta para el contenido de antocianinas de extracto de berenjena como función (a) temperatura y tiempo (concentración de etanol $70 \% \mathrm{v} / \mathrm{v}$ ); (b) Temperatura y concentración solvente (8 horas). Actividad antioxidante de extractos de cascara de berenjena 
La capacidad antioxidante de los extractos de cascara de berenjena, se observó que el modelo que explica significativamente $(p<0,05)$ la capacidad antioxidante de la cascara con respecto a las variables en estudio, concentración de solvente, tiempo y temperatura de extracción fue un modelo lineal. Los resultados del análisis de varianza (Tabla 3) muestran que variables tiempo y temperatura de extracción fueron significativos en la extracción de actividad antioxidante. Los coeficientes cuadráticos de las variables no fueron significativos para la actividad antioxidante. En cuanto a las interacciones de las variables solo fue significativa la interacción tiempo temperatura $(p<0.05)$.

Tabla 3. Análisis de Varianza de capacidad antioxidante

\begin{tabular}{ccccccc}
\hline Fuente & Suma de Cuadrados & Df & Cuadrados Medios & F Value & Prob > F & Significancia \\
\hline Modelo & 896,77 & 9,00 & 99,64 & 2,83 & 0.0226 & $\mathrm{~S}$ \\
A & 371,74 & 1,00 & 371,74 & 10,55 & 0.0037 & $\mathrm{~S}$ \\
B & 193,45 & 1,00 & 193,45 & 5,49 & 0.0286 & $\mathrm{~S}$ \\
C & 0,84 & 1,00 & 0,84 & 0,02 & 0.8786 & $\mathrm{NS}$ \\
$\mathrm{A}^{2}$ & 37,72 & 1,00 & 37,72 & 1,07 & 0.3122 & $\mathrm{NS}$ \\
$\mathrm{B}^{2}$ & 24,78 & 1,00 & 24,78 & 0,70 & 0.4108 & $\mathrm{NS}$ \\
$\mathrm{C}^{2}$ & 30,49 & 1,00 & 30,49 & 0,86 & 0.3624 & $\mathrm{NS}$ \\
AB & 180,01 & 1,00 & 180,01 & 5,11 & 0.0341 & $\mathrm{~S}$ \\
AC & 0,09 & 1,00 & 0,09 & 0,00 & 0.9605 & $\mathrm{NS}$ \\
BC & 0,06 & 1,00 & 0,06 & 0,00 & 0.9678 & $\mathrm{NS}$ \\
Residual & 775,53 & 22,00 & 35,25 & & & $\mathrm{NS}$ \\
Ajustes & 620,00 & 17,00 & 36,47 & 1,17 & 0.4683 & \\
Error & 155,53 & 5,00 & 31,11 & & & \\
Correlación Total & 1672,30 & 31,00 & & & &
\end{tabular}

El valor del coeficiente de determinación $\left(R^{2}\right)$ fue de 0,9169 indicando que el modelo obtenido es capaz de explicar el $91,69 \%$ de las variaciones en los contenidos de antocianinas. El modelo ajustado al contenido de antocianos presentó la siguiente ecuación con $\mathrm{t}=$ tiempo y $\mathrm{T}=$ temperatura

Capacidad antioxidante $=-3,51-4.04 \mathrm{t}+0.29 \mathrm{~T}-0.06 \mathrm{~T}$ * $\mathrm{t}$

Del modelo de la ecuación 2, se aprecia que el tiempo extracción ofrece un efecto superior con relación al provocado por la temperatura. La figura 2 muestra el gráfico de la superficie de respuesta descrita por el modelo.

Gisbert et al. (2011) reportó valores de sólidos en el rango de 4.02 a $4.18{ }^{\circ}$ Brix para diferentes variedades de berenjenas. Asimismo Muy et al. (2002), indicó valores promedios de sólidos solubles, pH y acidez de 3 . $4 ; 4.8$ y $0.15 \%$ respectivamente en berenjenas moradas de diferentes variedades, valores muy cercanos a los reportados en este estudio (Tabla 1). De esta manera la caracterización fisicoquímica de berenjena fucsia-morada y lila es similar a resultados obtenidos por otros autores (Muy et al. 2002 Brandão et al., 2003; Moretti y Pineli, 2005; Prohens et al., 2007; Gajewski et al., 2009).

Estos resultados de contenido de fenoles son similares a los reportados por Luthria et al. (2010), quien determinó contenido de compuestos fenólicos de berenjena negra campana (Americana) y Millionaire (Japonesa) de $9.88 \mathrm{mg}$ de acido gálico g-1 y $13.64 \mathrm{mg} \mathrm{g}-1$ (Base seca) respectivamente. Raigón et al. (2008), reporta contenido de fenoles en un rango de $34.46 \mathrm{mg} / 100 \mathrm{~g}$ a $60.70 \mathrm{mg} / 100 \mathrm{~g}$ en diferentes variedades de berenjena. Sin embargo se han reportado valores de fenoles totales más altos en berenjena de diferentes variedades; Gajewski et al. (2009), reportó fenoles totales de 0.83mg g-1 de pulpa fresca de berenjena morada negra; Nisha et al. (2009), mostró contenido de fenoles para berenjena morada, grande y de forma redondeada del $49.02 \mathrm{mg} / 100 \mathrm{~g}$. mientras que el contenido de fenoles para berenjena morada pequeña fue de $106.98 \mathrm{mg} / 100 \mathrm{~g}$. Gisbert et al. (2011) reportó contenido de fenoles totales entre 456 y 550 $\mathrm{mg} \mathrm{kg-1}$ de pulpa de berenjenas con resultantes del cruzamiento genético de varios híbridos. 


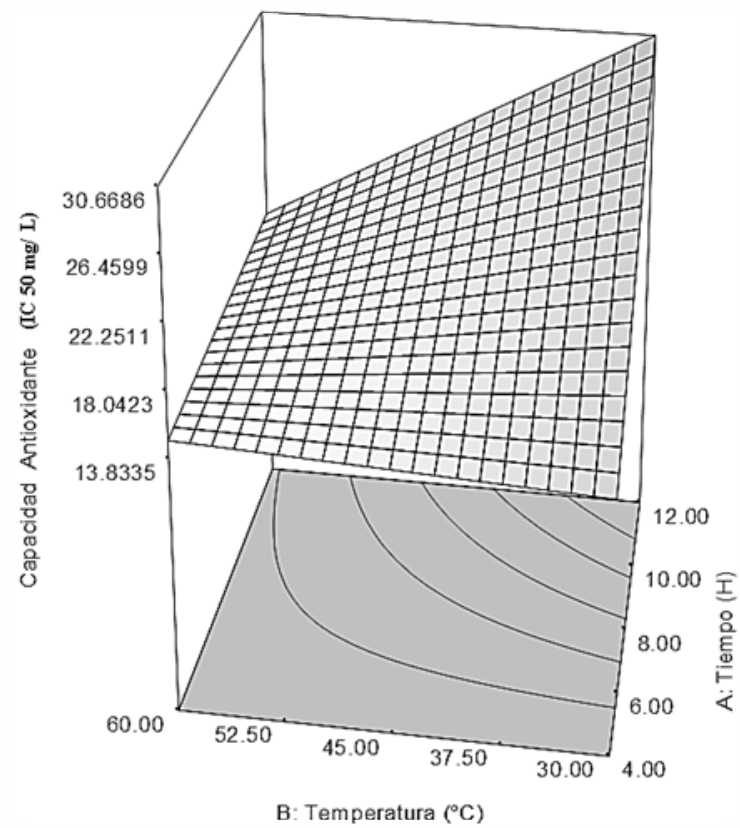

Fig. 2. Superficie de respuesta para actividad antioxidante de extracto de berenjena Morada como función de la temperatura y tiempo (a concentración de etanol $70 \% \mathrm{v} / \mathrm{v}$ ).

Muchos autores han investigado fenoles de berenjena de diferentes variedades, sin embargo los resultados no son similares, debido a las diferencias existentes en las condiciones en que se desarrollaron los experimentos, y por el gran número de variables que influyen en la extracción de estos compuestos (Akanitapichat et al., 2010; Luthria et al., 2010; Kwon et al., 2008). Jung et al. (2011) obtuvo resultados similares de contenidos de antocianinas en pulpa de berenjena $(2.29 \mathrm{mg} / 100 \mathrm{~g})$. Contenidos más bajos de antocianinas en pulpa de berenjena fueron reportados por Nisha et al. (2009), el cual obtuvo contenidos de antocianos de $0.530(\mathrm{mg} / 100 \mathrm{~g})$ y $0.756(\mathrm{mg} / 100 \mathrm{~g})$ en frutos de berenjenas moradas de diferentes tamaños. Según Gajewski et al. (2009), reporto que el contenido de antocianinas en la cascara de berenjena fue de $3.2 \mathrm{mg} \mathrm{g}^{-1}$ para berenjena fresca variedad "Scorpio" morada negra.

En cuanto a la obtención de antocianinas, se observó que aumentar la temperatura se produce un incremento en el rendimiento extracción del pigmento, probablemente debido a que se incrementa la solubilidad de las antocianinas y por ende el coeficiente de difusión del disolvente líquido en la matriz sólida favorece la cinética de desorción de los compuestos de la matriz (Cacace y Mazza, 2003). Sin embargo las altas temperaturas influyen marcadamente en la destrucción de las antocianinas, debido a que pueden causar la pérdida del azúcar glicosilante en la posición 3 de la molécula y la apertura de anillo pirano con la consecuente producción de chalconas incoloras (Falcão et al., 2003; Falcão et al., 2008; Garzon, 2008). El tiempo de extracción es un importante factor en la extracción de antocianos; sin embargo después de un tiempo excesivo de podría no ser significativo la extracción de pigmentos, la ley de difusión de Fick, predice que después de cierto tiempo, habrá un equilibrio final entre el soluto de la matriz solida y el solvente de extracción (Silva et al., 2007).

Los resultados de la optimización del modelo muestran que las condiciones de extracción con $50 \%$ de solvente (Etanol), tiempo de 4 horas y temperatura de $30{ }^{\circ} \mathrm{C}$ presentó los mayores contenidos de antocianinas de $62 \mathrm{mg} / 100 \mathrm{~g}$ en cascara de berenjena. Todaro et al. (2009), obtuvo un resultado similar de contenido de antocianinas de $76.44 \mathrm{mg} / 100 \mathrm{~g}$ de cascara. Con los resultados obtenidos, utilizando el modelo de superficie de respuesta aplicado, se pueden apreciar cómo el tiempo y la temperatura del proceso influyen significativamente en la capacidad antioxidante de las muestras de extracto de berenjena y es evidente que a medida que el factor tiempo se incrementan, aumenta la capacidad antioxidante, posiblemente debido a que a mayor tiempo de contacto de la matriz con el solvente, mayor extracción de compuestos fenólicos y antocianos, los cuales son responsables de la capacidad antioxidante (2002; Zheng et al., 2011).

Asimismo los tratamientos de extracción a altas temperaturas y tiempos prolongados, causan una disminución en la capacidad antioxidante (Pérez et al., 2008). Este hecho puede ser explicado debido a que el proceso de extracción a tiempos prolongados y a altas temperaturas puede dar lugar a la oxidación de 
compuestos fenólicos, por la luz, exposición al oxígeno y a la degradación (Spigno et al., 2007). Además la altas temperaturas de extracción podrían producir la destrucción térmica de los compuestos fenólicos y consecuentemente causar la reducción en la capacidad antioxidante del extracto (Chew et al., 2011). Los resultados de capacidad antioxidante en extractos de cascara de berenjena con las diferentes variables de estudio muestran una capacidad antioxidante en un rango de IC50 de 8.42 a $37.28 \mathrm{mg} / \mathrm{L}$. Akanitapichat et al., (2010), encontró resultados mayores de capacidad antioxidante al reportar IC50 de $53.18 \mathrm{mg} / \mathrm{L}$ en fruto de berenjena morada, sin embargo hay que tener en cuenta que éste autor evalúo la capacidad antioxidante incluyendo la pulpa del fruto.

\section{CONCLUSIONES}

Los factores de extracción de antocianinas tiempo (4-12 horas), temperatura (30-60 $\mathrm{C}$ ) y la concentración de solvente (50-90\%) afectan linealmente el contenido de antocianinas extraídas de la cascara de berenjena, siendo la temperatura la que ejerce un efecto cuadrático. La optimización del modelo muestra que las condiciones de extracción con $50 \%$ de solvente (Etanol), tiempo de 4 horas y temperatura de $30^{\mathrm{a}} \mathrm{C}$ presentó los mayores contenidos de antocianinas de $62 \mathrm{mg} / 100 \mathrm{~g}$ en cascara de berenjena. La interacción tiempo de extracción y temperatura afecta la capacidad antioxidante de extractos de berenjena, de forma tal que su incremento provoca una disminución significativa en la actividad antioxidante. Los resultados de capacidad antioxidante en extractos de cascara de berenjena con las diferentes variables de estudio muestran una capacidad antioxidante en un rango de IC50 de 8.42 a $37.28 \mathrm{mg} / \mathrm{L}$.El contenido de antocianinas en pulpa de berenjena Fucsia-Morada y Lila representa menos del $1 \%$ del total de fenoles encontrados en la pulpa del fruto.

\section{AGRADECIMIENTOS}

Los autores desean expresar su agradecimiento Al Departamento Administrativo de Ciencia, Tecnología e Innovación "Colciencias" y a su programa de becas de Jóvenes Investigadores e Innovadores "Virginia Gutiérrez de Pineda" por el apoyo financiero brindado para la ejecución de esta investigación.

\section{REFERENCIAS}

Akanitapichat, P., K. Phraibung, K. Nuchklang y S. Prompitakkul, Antioxidant and hepatoprotective activities of five eggplant varieties. Food Chemestry Toxicology.48: 3017-3021. (2010).

Botelho, F., L. Eneas, G. Cesar, C. Bizzotto, E. Tavares, F. Oliveira, M. Gloria, M. Silvestre, R. Arantes y Alvarez, J., Effects of eggplant (Solanum melongena) on the atherogenesis and oxidative stress in LDL receptor knock out mice (LDLR). Food Chemestry Toxicology. 42: 1259-1267. (2004).

Brandão, J., R. Goto, M. Da Silva,H. Silva, J. De Bastos, Influência da Enxertia na Qualidade de Frutos da Berinjela Sob. Cultivo Protegido. Agronomia. 37 (1):86 - 89, (2003)

Cacace, J. E. y G. Mazza, Optimization of extraction of anthocyanins from black currants with aqueous ethanol. Journal Food Science. 68:240-248, (2003)

Chew, K., M. Khoo, S. Ng, Y. Thoo, W. Wan Aida, y C. Ho, Effect of ethanol concentration, extraction time and extraction temperature on the recovery of phenolic compounds and antioxidant capacity of Orthosiphon stamineus extracts. International Food Research Journal. 18(4):1427-1435,(2011).

Falcão, L., A. Falcão, E. Gris y M. Bordignon, Spectrophotometric study of the stability of anthocyanins from Cabernet Sauvignon grape skins in a model system. Brazilian Journal of Food Technology. 11(1):63-69. (2008).

Falcão, L., Estabilidade de antocianinas extraídas de uvas Cabernet Sauvignon (Vitis vinifera L.) em solução tampão, bebida isotônica e iogurte. Mestre em Ciência dos Alimentos. Universidade Federal de Santa Catarina. Brasil. (2003).

Gajewski, M., K. Katarzyna y M. Bajer, The Influence of Postharvest Storage on Quality Characteristics of Fruit of Eggplant Cultivars. Not. Bot. Hort. Agrobot. 37 (2):200-205, (2009).

Garzón, G. Las antocianinas como colorantes naturales y compuestos bioactivos: revisión. Acta biol. Colombia. 13 (3):27-36, (2008). 
Gisbert, C., J. Prohens, M. Raigón, J. Stommel y F. Nueza, Eggplant relatives as sources of variation for developing new rootstocks: Effects of grafting on eggplant yield and fruit apparent quality and composition. Scientia Horticulturae. 128: 14-22, (2001).

Giusti, M. y R. Wrolstad, Characterization and Measurement of Anthocyanins by UV-Visible Spectroscopy. Current Protocols in Food Analytical Chemistry. F1.2.1- F1.2.13, (2001).

Jung, E., M. Bae, E. Jo., Y. Jo, y S. Lee, Antioxidant activity of different parts of eggplant. Journal of Medicinal Plants Research. 5(18):4610-4615, (2011).

Kong, J., L. Chia, N. Goh, T. Chia, y R. Brouillard, Analysis and biological activities of anthocyanins. Phytochemistry. 64: 923-933, (2003).

Kwon, Y., E. Apostolidis, y K. Shetty, In vitro studies of eggplant (Solanum melongena) phenolics as inhibitors of key enzymes relevant for type 2 diabetes and hypertension. Bioresour Technology. 99: 29812988, (2008).

Hongyan, Li., Z. Deng, H. Zhu, Hu. Chanli, R. Liu, Y. Christopher y R. Tsao, Highly pigmented vegetables: Anthocyanin compositions and their role in antioxidant activities. Food Research International 46:250-259, (2012).

Longo, L. y G. Vasapollo, Extraction and identification of anthocyanins from Smilax aspera L. berries. Food Chemistry. 94:226-231, (2006).

Luthria, D., A. Singh, T. Wilson, G. Banuelos, y B. Vinyard, Influence of conventional and organic agricultural practices on the phenolic content in eggplant pulp: Plant-to-plant variation. Food Chemistry. 121:406-411, (2010).

Martínez, B. Evaluación de la estabilidad, las propiedades antioxidantes y antimicrobianas de dos extractos de plantas aromáticas limoncillo (Cymbopogon citratus) y cúrcuma (Curcuma longa).Tesis Ingeniero de Alimentos. Universidad de Córdoba. Colombia, (2009).

Moretti, C. Y L. Pineli, Qualidade Química E Física De Berinjelas Submetidas A Diferentes Tratamentos Pós-Colheita. Ciênc. Tecnol. Aliment. 25(2):339-344, (2005).

Muy, D., J. Siller, R. García y M. Báez, Caracterización poscosecha de Berenjenas producidas en Sinaloa, México. Revista Chapingo Serie Horticultura. 8(2):171-181, (2002).

Nisha, P., P. Nazar y P. Jayamurthy, A comparative study on antioxidant activities of different varieties of Solanum melongena. Food Chemestry Toxicology. 47: 2640-2644, (2009).

Noda, Y., T. Kneyuki, K. Igarashi, A. Mori y L. Packer, Antioxidant activity of nasunin, an anthocyanin in eggplant peels. Toxicology. 148: 119-123, (2000).

Pérez, J., S. Arranz, M. Tabernero, M. Díaz, J. Serrano, I. Goñi y F. Saura, Updated methodology to determine antioxidant capacity in plant foods, oils and beverages: Extraction, measurement and expression of results. Food Research International. 41: 274-285, (2008).

Poo, B., Concentración de Antocianinas en Jugo de Cranberries (Vaccinium macrocarpon Ait.) mediante Nanofiltración. Tesis de Licenciado en Ciencias de los Alimentos. Universidad Austral de Chile. Chile. (2005).

Prohens, J., A. Rodríguez, M. Raigón y F. Nuez, Total Phenolic Concentration and Browning Susceptibility in a Collection of Different Varietal Types and Hybrids of Eggplant: Implications for Breeding for Higher Nutritional Quality and Reduced Browning. J. Amer. Soc. Hort. 132(5):638-646, (2007).

Raigón, M., J. Prohens, J. Muñoz y F. Nuez, Comparison of eggplant landraces and commercial varieties for fruit content of phenolics, minerals, dry matter and protein. Journal of Food Composition and Analysis. 21: 370-376, (2008).

Severo, J., S. Galarça, R. Aires, R. Cantillano, C. Rombaldi y J. Silva, Avaliação de compostos fenólicos, antocianinas, vitamina $C$ e capacidade antioxidante em mirtilo armazenado em atmosfera controlada. Braz. Journal Food Technology. 2: 65-70, (2009). 
Silva, M., H. Rogez y Y. Larondelle, Optimization of extraction of phenolics from Inga edulis leaves using response surface methodology. Separation and Purification Technology. 55 (3): 381-387, (2007).

Singh, A., D. Luthria, T. Wilson, N. Vorsa, V. Singh, G. Banuelos y S. Pasakdee, Polyphenols content and antioxidant capacity of eggplant pulp. Food Chemistry. 114: 955-961, (2009).

Spigno, G., L. Tramelli y D. De Faveri, Effects of extraction time, temperature and solvent on concentration and antioxidant activity of grape marc phenolics. Journal. Food Engineering. 81: 200-208, (2007).

Todaro, A., F. Cimino, P. Rapisarda, A. Catalano, R. Barbagallo, y G.Spagna, Recovery of anthocyanins from eggplant peel. Food Chemistry. 114: 434-439, (2009).

Trejo, M.. Ma. A. y S. P. Bustamantel, Evaluación de capacidad antioxidante y determinación de fenoles totales para frutos. Universidad Autónoma de México. P. 16, (2002).

Zheng, N., Z. Wang, F. Chen y J. Lin, Evaluation to the antioxidant activity of total flavonoids extract from Syzygium jambos seeds and optimization by response surface methodology. Journal Pharm Pharmacol. (21):2411-2419, (2011). 
